\title{
Energy-Efficient Routing Protocol for Wireless Sensor Networks Based on I mproved Grey Wolf Optimizer
}

\author{
Xiaoqiang Zhao ${ }^{1,2}$, Hui Zhu ${ }^{1,2}$, Slavisa Aleksic ${ }^{3}$, Qiang Gao ${ }^{1}$ \\ ${ }^{1}$ School of Communication and Information, Xi'an University of Posts and Telecommunications \\ Xi'an, Shaanxi, 710121, P.R. China \\ ${ }^{2}$ Shaanxi Key Laboratory of Information Communication Network and Security \\ Xi'an, Shaanxi, 710121, P.R. China \\ [e-mail: zxq7703@126.com] \\ ${ }^{3}$ Institute of Communications Engineering, Leipzig University of Telecommunications \\ Leipzig, 04277, Germany \\ [e-mail: aleksic@hft-leipzig.de] \\ *Corresponding author: Hui Zhu
}

Received November 6, 2017; revised January 1, 2018; accepted January 10, 2018; published June 30, 2018

\begin{abstract}
To utilize the energy of sensor nodes efficiently and extend the network lifetime maximally is one of the primary goals in wireless sensor networks (WSNs). Thus, designing an energy-efficient protocol to optimize the determination of cluster heads (CHs) in WSNs has become increasingly important. In this paper, we propose a novel energy-efficient protocol based on an improved Grey Wolf Optimizer (GWO), which we refer to as Fitness value based Improved GWO (FIGWO). It considers a fitness value to improve the finding of the optimal solution in GWO, which ensures a better distribution of CHs and a more balanced cluster structure. According to the distance to the $\mathrm{CHs}$ and the BS, sensor nodes' transmission distance are recalculated to reduce the energy consumption. Simulation results demonstrate that the proposed approach can prolong the stability period of the network in comparison to other algorithms, namely by $31.5 \%$ in comparison to SEP, and even by $57.8 \%$ when compared with LEACH protocol. The results also show that the proposed protocol performs well over the above comparative protocols in terms of energy consumption and network throughput.
\end{abstract}

Keywords: wireless sensor networks (WSNs), energy-efficiency, Gray Wolf Optimizer (GWO), balanced cluster structure, network lifetime

This work was supported by the Science and Technology Co-ordination Innovation Program of Shaanxi Province (No.: 2016KTCQ01-26), Xi'an Science and Technology Project - Lanhuan Smart Green Cloud and Public Service Platform Research and Development (No.:2017084CG/RC047) and the Science and Technology Innovation Team of Shaanxi Province for Broadband Wireless and Application (No.: 2017KCT-30-02). 


\section{Introduction}

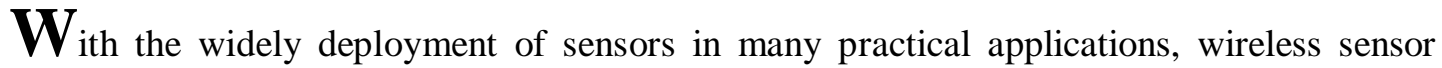
networks (WSNs) are gaining more and more attention. In WSN, the sensor nodes are scattered to monitor humidity, temperature, pressure, etc. However, their computing power, battery capacity, and communication distance are very limited [1]. Besides, in many WSN scenarios, sensor nodes are deployed in harsh environments, which makes the replacement and recharge of failed nodes difficult and expensive. Moreover, once the sensor nodes are deployed in the area of interest, they keep operating until they run out of power. Therefore, how to design an energy-efficient routing protocol for WSN is one of the greatest challenges in prolonging the network's lifespan [2].

Clustering based hierarchical routing protocol is particularly effective in improving energy efficiency and increasing network longevity by minimizing overall transmission distance and balancing energy consumption among the nodes during the network lifetime [3]. The sensing area is partitioned into multiple clusters using the clustering protocol. In each cluster, a certain node will perform the task of a leader node, the so called cluster head $(\mathrm{CH})$. $\mathrm{CH}$ is the center of the cluster structure, its role is to communicate with the cluster members (CM), collect data from CMs, and send them to the base station (BS) after the fusion process. Thus, how to optimize the selection of $\mathrm{CHs}$ and obtain a better distributed cluster structure are the most important problem in clustering-based routing protocols.

The remaining part of the paper is organized as follows. Section 2 describes related work in this area. Section 3 introduces the GWO method. Section 4 gives the details of the proposed algorithm. Section 5 presents simulation results and discussions. Finally, Section 6 concludes the paper.

\section{Related Work}

Numerous energy-efficient routing protocols have been designed on the basis of clustering structure [4]. In the following, we give a brief review of some selected clustering-based protocols, which are related to the current work. LEACH (Low Energy Adaptive Clustering Hierarchy) [5] was the first hierarchal protocol for homogeneous WSNs based on clustering. In LEACH, nodes are organized into clusters and there exists a single $\mathrm{CH}$ for each cluster. Data from all the other nodes within a cluster is transferred to the respective $\mathrm{CH}$. $\mathrm{CH}$ consumes additional energy in comparison to the normal nodes. However, the random selection of CHs leads to excessive energy consumption of the $\mathrm{CH}$. This is mainly because of the need for a higher transmission power to overcome the longer distance. Therefore, the node consumes more energy and dies quickly, which leads to a decrease of the network's lifespan. The prolong stable election protocol (P-SEP) [6] was an improvement of the previously proposed SEP protocol. It proposes to prolong the stable period of fog-supported sensor networks by maintaining balanced energy consumption. Two-level nodes' heterogeneities are considered in P-SEP: advanced and normal nodes. Both the advanced and normal nodes have the same opportunity to become $\mathrm{CH}$. This approach shows improved performance in network lifetime. Another hierarchical routing algorithm was proposed in Ref. [7] for large-scale mobile ad hoc networks (LMANET). The protocol utilizes table-driven and on-demand routing to search dominant set of nodes, which composed by link expiration tiem and node's relative degree to establish the intra-/inter-communication paths in LMANET. In the work presented by Ray et 
al. [8], a midpoint approach based on the K-means algorithm was proposed. The nodes are sorted into different sets in accordance with their distance to the BS, thereby ensuring that CHs are uniformly distributed over the network. Ma et al. [9] proposed a clustering algorithm based on the residual energy of the node and the network. Nodes with less residual energy have lower chances to be selected as $\mathrm{CH}$. The lifetime, throughput and energy efficiency of the network are enhanced through this technique. In Ref. [10], Park et al. presented an improved $\mathrm{CH}$ selection method based on K-means. The initial centroids are selected randomly, which results in different kinds of clusters in different runs. The residual energy of nodes is considered as a parameter of $\mathrm{CH}$ selection, but does not specify any proper estimation.

As WSN routing becomes more challenging and complex, researchers have proposed many bio-inspired meta-heuristic algorithms. Ahmadi et al. [11] proposed an effective technique for preserving k-coverage and reliability of data with logical fault tolerance. In this algorithm, nodes' residual energy and their neighbors' information are known to each node. All network nodes are divided into coverage and communicative nodes, and then some nodes are re-categorized as clustering and dynamic nodes. The method shows good energy consumption efficiency. In the work by Al-Aboody et al. [12], a multi-level hybrid clustering routing protocol (MLHP) based on the GWO was proposed. The authors implemented the GWO algorithm at level two without changing the original algorithm. This algorithm is able to achieve better performance in network's energy efficiency, lifetime, and stability period. However, this mechanism has a very long un-stability period, which results in an unstable data transmission. Ref. [13] presented an efficient cover set algorithm based on the Imperialist Competitive Algorithm (ICA). Considering the advantage of ICA, the proposed algorithm selects the sensor nodes in different cover sets and generates the cover sets to monitor all deployed targets. This approach performs well in terms of extending network lifetime.

In this paper, we propose a fitness value based IGWO (FIGWO), which has the advantages of further prolonging the network lifetime. Several techniques are used in FIGWO for the selection of CHs: (1) instead of choosing initial CHs randomly, we apply a distance method for initial CHs selection; (2) FIGWO approach considers a fitness value as the weight in GWO, so that the nodes with higher fitness value are more likely to be selected as the CHs; (3) if the distance between a $\mathrm{CM}$ and its corresponding $\mathrm{CH}$ is greater than its distance to the $\mathrm{BS}$, this $\mathrm{CM}$ will send its sensed information to the BS directly. Thus, a reduction in data transmission to related $\mathrm{CH}$ energy consumption can be achieved by keeping the communicating distance between CHs and the BS as well CMs and CHs short. As a result, the network lifetime is enhanced through the above mechanism.

\section{Grey Wolf Optimizer}

GWO is inspired by the social hierarchy and hunting behavior of grey wolf packs [14]. Wolves are categorized into four types. In the hunting (optimization), the $\alpha, \beta$ and $\delta$ wolves are responsible to evaluate the prey location (optimum), the rest wolves calculate the distance between themselves and the prey, then complete the encirclement of the prey. Here are the definitions with great importance in the GWO.

Definition 1: Location of the wolves

$$
\begin{aligned}
& \overrightarrow{X^{t+1}}=\overrightarrow{X_{p}^{t}}-\vec{A} \times \vec{D} \\
& \vec{A}=2 \times \vec{\alpha} \times \overrightarrow{r_{1}}-\vec{\alpha}
\end{aligned}
$$


where $\overrightarrow{X^{t+1}}$ is the wolves' position in the $(t+1)^{t h}$ iteration, $\overrightarrow{X_{p}^{t}}$ is the prey's position in the $t^{t h}$ iteration. $\vec{A}$ is the convergence factor. $\vec{\alpha}$ is linearly decreased from 2 to 0 over the course of the iterations, and $\vec{r}_{1}$ is random vectors in the range $[0,1] . \vec{D}$ is the distance between the wolves and the prey. It is mathematically modeled by the following equations:

$$
\begin{gathered}
\vec{D}=\left|\vec{C} \times \overrightarrow{X_{p}^{t}}-\overrightarrow{X^{t}}\right| \\
\vec{C}=2 \times \overrightarrow{r_{2}}
\end{gathered}
$$

where $\vec{X}_{p}^{t}$ is the prey's position in the $t^{\text {th }}$ iteration, and $\vec{X}^{t}$ is the wolf's position in the $t^{\text {th }}$ iteration, and $\vec{C}$ is the coefficient vectors calculated in (4), $\overrightarrow{r_{2}}$ is a random vector between 0 and 1.

Definition 2: Location of the prey

Among the wolves, the $\alpha, \beta$ and $\delta$ wolves are closest to the prey, and they have the most extensive experience, so they can determine where the prey is located. Therefore, the location of the prey can be calculated as:

$$
\overrightarrow{X_{p}^{t+1}}=\frac{\overrightarrow{X_{\alpha}^{t+1}+}+\overrightarrow{X_{\beta}^{t+1}}+\overrightarrow{X_{\delta}^{t+1}}}{3}
$$

where $\overrightarrow{X_{\alpha}^{t+1}}, \overrightarrow{X_{\beta}^{t+1}}$ and $\overrightarrow{X_{\delta}^{t+1}}$ are the position of $\alpha$ wolf, $\beta$ wolf and $\delta$ wolf in the $(t+1)^{t h}$ iteration, respectively. They are all calculated with accordance to equation (1).

In the algorithm presented in this paper, we assume that grey wolves are the sensor nodes and the prey is the $\mathrm{CH}$.

\section{The Proposed Algorithm}

In this section, the fitness value-based improved GWO algorithm (FIGWO) is presented in detail. The main objective of the proposed study is to enhance the network lifetime and minimize the energy consumption of sensor nodes. Similar to the traditional clustering routing protocols in WSNs, the FIGWO defines rounds and each round is divided into cluster building phase and data transmission phase.

\subsection{Network Model}

In the network model, the following assumptions are made:

(1) The BS is located in the center of the sensing area and is externally powered.

(2) All nodes are randomly distributed, and once deployed, the nodes are not changing their positions.

(3) All sensors nodes are homogeneous and have the function of data fusion.

(4) After deployment, the BS knows all the information of all sensor nodes. And the algorithm for the selection of CHs is executed in the BS. 


\subsection{Energy Model}

The energy model used here is similar to that presented in [12]. To transmit a $l$-bit long data packet over the distance $d$, the required energy is:

$$
E_{T X}(l, d)=\left\{\begin{array}{lll}
l \times E_{\text {elec }}+l \times \varepsilon_{f s} \times d^{2}, & \text { if } & d<d_{0} \\
l \times E_{\text {elec }}+l \times \varepsilon_{m p} \times d^{4}, & \text { if } & d>d_{0}
\end{array}\right.
$$

where $E_{T X}$ is the transmitted energy, $E_{\text {elec }}$ is the energy dissipated per bit in the transmitter or receiver circuit. $\varepsilon_{f s}$ and $\varepsilon_{m p}$ depend on the transmitter amplifier model. If the distance between the transmitter and the receiver is less than a threshold $d_{0}$, the free space model is used; otherwise, the multi-path model is used. $d_{0}$ is usually calculated as:

$$
d_{0}=\sqrt{\frac{\varepsilon_{f s}}{\varepsilon_{m p}}}
$$

On the other side, the energy consumption for the receiver to receive a $l$-bit long packet is calculated as follows:

\subsection{The Proposed Algorithm}

$$
E_{T X}(l)=l \times E_{\text {elec }}
$$

The FIGWO defines rounds and each round is divided into cluster building phase and data transmission phase.

\section{Cluster Building Phase}

The CHs are important nodes in the clustering routing protocols because they collect, process, and transmit the packets originating from the CMs in the same cluster. The pseudocode for cluster building is presented in Table 1.

Table 1. The pseudocode for clustering building phase

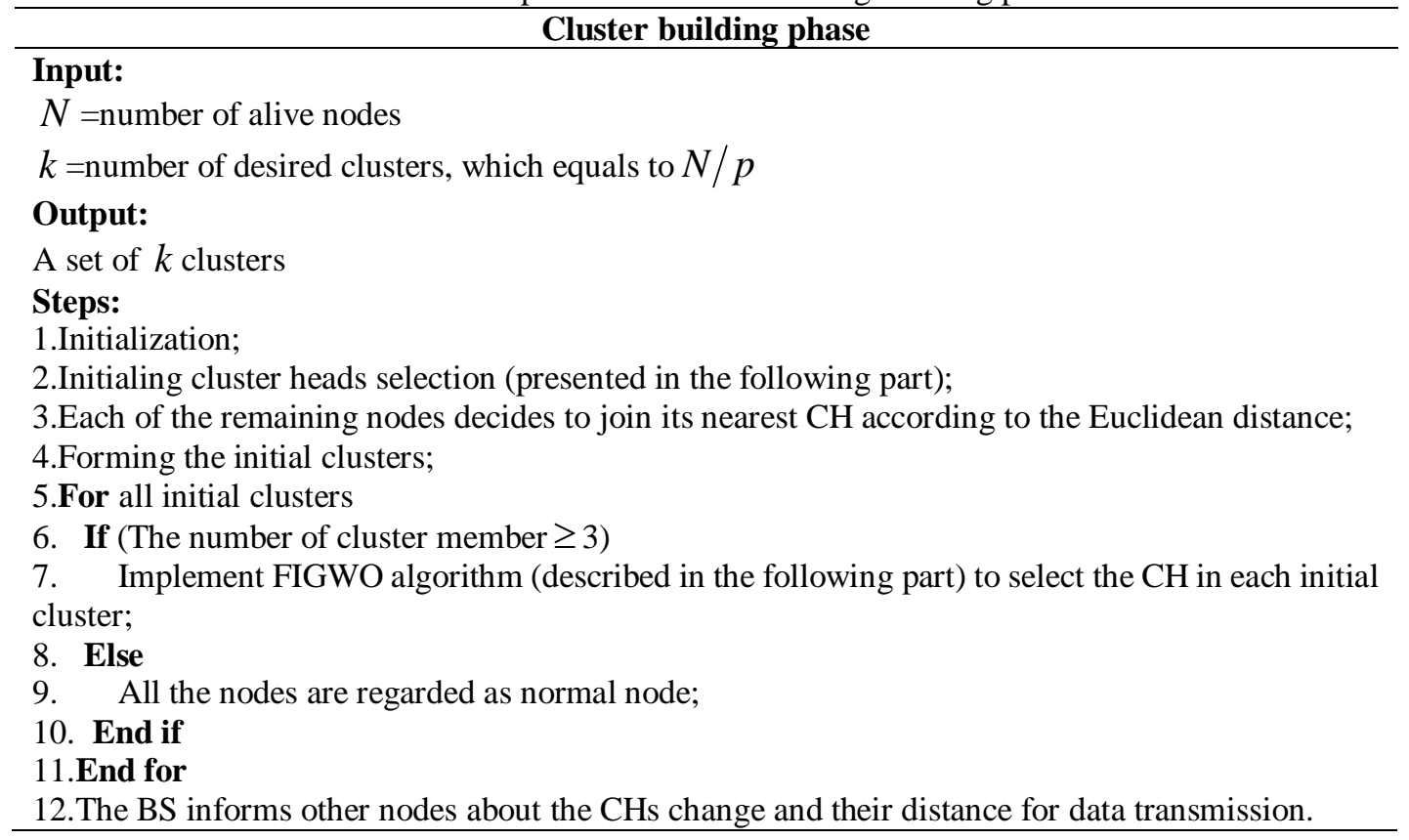


The initial clusters are selected by the following rules. The set of alive sensor nodes is partitioned into $k$ equal subsets according to the fitness value. In each set, the sensor node that is nearest to the middle point is taken as the initial cluster head. The node's fitness value is calculated according to its distance to the BS, and its residual energy:

$$
F= \begin{cases}a \times \frac{E_{r}}{E_{i}}+(1-a) \times\left(\frac{d_{M A X}-d}{d_{M A X}-d_{M I N}}\right), & E_{r}>0 \\ 0 \quad, & E_{r} \leq 0\end{cases}
$$

where $a$ is a coefficient which indicates the contribution between $E_{r}$ and $d$ in the fitness function $F . E_{r}$ represents the residual energy a alive node, while $E_{i}$ is the initial energy of a node; $d$ is the distance from the node to the BS; $d_{\text {MAX }}$ and $d_{\text {MIN }}$ are the maximum and the minimum distance between a sensor node and the BS, respectively.

The FIGWO algorithm is used to select CHs in initial clusters. Based on GWO and equation (9), the fitness value is calculated and used as the weights to determine the final position of the optimal solution which fully takes the node's current state into consideration. Consequently, the new position of prey is computed as follows:

$$
\begin{aligned}
& X_{p}^{t+1}=F_{w \alpha} \overrightarrow{X_{\alpha}^{t+1}}+F_{w \beta} X_{\beta}^{t+1}+F_{w \delta} X_{\delta}^{t+1} \\
& F_{w \alpha}=\frac{F_{\alpha}}{F_{\alpha}+F_{\beta}+F_{\delta}}, F_{w \beta}=\frac{F_{\beta}}{F_{\alpha}+F_{\beta}+F_{\delta}}, F_{w \delta}=\frac{F_{\delta}}{F_{\alpha}+F_{\beta}+F_{\delta}}
\end{aligned}
$$

where $F_{w \alpha}, F_{w \beta}$ and $F_{w \delta}$ represent the new weights of $\alpha$ wolf, $\beta$ wolf and $\delta$ wolf, respectively, which are calculated by equation (11). And $F_{\alpha}, F_{\beta}$ and $F_{\delta}$ are the best three fitness values. The details are showed in Table 2.

Table 2. The pseudocode of FIGWO

\begin{tabular}{l}
\hline \multicolumn{1}{c}{ FIGWO } \\
\hline 1: while $t<t_{\max }$ do \\
2: $\quad$ for each node in initial cluster do \\
3: $\quad$ Compute the fitness value according to (9). \\
4: $\quad$ Select the leader node $X_{\alpha}, X_{\beta}, X_{\delta}$ according to the best three fitness values. \\
5: $\quad$ Update the position of the prey using (10). \\
6: end for \\
7: end while \\
8: Select the node which has not been selected as $\mathrm{CH}$ in the latest $1 / p$ rounds. \\
\hline
\end{tabular}

\section{Data transmission phase}

The transmission phase mainly consists of two parts: the data transmission from the CMs to the $\mathrm{CH}$ and the data forwarding from the $\mathrm{CH}$ to the $\mathrm{BS}$. To reduce the transmission distance, if a sensor node's distance to the $\mathrm{CH}$ is shorter than the distance to the BS, then the sensor node sends its sensed information to its corresponding $\mathrm{CH}$. Otherwise, the sensor node directly sends its sensed data to the BS. Then, the $\mathrm{CH}$ collects the data and forwards the received packets in an aggregated form to the BS periodically. It is a single hop communication between the $\mathrm{CH}$ and the BS. Here, the transmission distance, $d$, and the transmitted packet size, $l$, are two factors that influence the energy consumption of the packet transmission phase. 


\subsection{Comparison of clustering algorithms}

Table 3 presents a comparison between several selected clustering-based algorithms and the proposed FIGWO algorithm. As can be seen from the table, FIGWO algorithm combines the heuristic algorithm and the clustering-based algorithm, likewise, considers the residual energy and location information of the nodes.

Table 3. Comparison of relevant algorithms

\begin{tabular}{|c|c|c|c|c|c|c|c|}
\hline Protocol & Ref. & Node type & $\begin{array}{c}\text { Inter-cluster } \\
\text { topology }\end{array}$ & $\begin{array}{c}\text { Location } \\
\text { awareness }\end{array}$ & $\begin{array}{c}\text { Energy } \\
\text { awareness }\end{array}$ & $\begin{array}{c}\text { CH } \\
\text { election }\end{array}$ & $\begin{array}{c}\text { Using } \\
\text { heuristic } \\
\text { algorithm }\end{array}$ \\
\hline LEACH & $\begin{array}{l}\text { Heinzelman } \\
\text { et al. (2000) }\end{array}$ & Homogeneous & Direct & $\begin{array}{l}\text { Not } \\
\text { required }\end{array}$ & $\begin{array}{l}\text { Not } \\
\text { required }\end{array}$ & Random & No \\
\hline $\begin{array}{c}\text { CARED } \\
\text { R }\end{array}$ & $\begin{array}{l}\text { Ma et al. } \\
\text { (2013) }\end{array}$ & Homogeneous & Multi-hop & $\begin{array}{l}\text { Not } \\
\text { required }\end{array}$ & Required & Deterministic & No \\
\hline MLHP & $\begin{array}{l}\text { Al-Aboody } \\
\text { et al. (2016) }\end{array}$ & Heterogeneous & Multi-hop & $\begin{array}{l}\text { Not } \\
\text { required }\end{array}$ & $\begin{array}{l}\text { Not } \\
\text { required }\end{array}$ & Deterministic & Yes \\
\hline P-SEP & $\begin{array}{l}\text { Naranjo et } \\
\text { al. (2017) }\end{array}$ & Heterogeneous & Direct & Required & Required & Deterministic & No \\
\hline FIGWO & I & Homogeneous & Direct & Required & Required & Deterministic & Yes \\
\hline
\end{tabular}

\section{Simulation results}

To evaluate the performance of the FIGWO algorithm, extensive simulations were carried out. Both the network model and the FIGWO algorithm were implemented using MATLAB. The results obtained for the FIGWO algorithm are compared to those obtained using the SEP and LEACH algorithms under the same circumstances. For this purpose, we also implemented those algorithms. The simulation was ran considering the following two scenarios: node numbers $N=\{100,200\}$,sensing areas $M=\{100 \times 100,200 \times 200\}$. The main simulation parameters are listed in Table 4.

Table 4. Simulation Parameters

\begin{tabular}{ll}
\hline Parameter & value \\
\hline Network Size & $\left\{100^{2}, 200^{2}\right\}\left(\mathrm{m}^{2}\right)$ \\
Number of Sensor nodes & $\{100,200\}$ \\
Portion of CHs & $p=0.1$ \\
Packet Size & $l=4000 \mathrm{bits}$ \\
Data Aggregation Energy Cost & $\mathrm{E}_{\mathrm{DA}}=50 \mathrm{~nJ} / \mathrm{bit}$ \\
Transmitter/Receiver & $\mathrm{E}_{\text {elec }}=50 \mathrm{~nJ} / \mathrm{bit}$ \\
Transmitter Amplifier (free space) & $\varepsilon_{f \mathrm{~s}}=10 \mathrm{pJ} /\left(\mathrm{bit} \cdot \mathrm{m}^{2}\right)$ \\
Transmitter Amplifier (multi-path space) & $\varepsilon_{m p}=0.0013 \mathrm{pJ} /\left(\mathrm{bit} \cdot \mathrm{m}^{4}\right)$ \\
Coefficient of Fitness function & $\mathrm{a}=0.2$ \\
\hline
\end{tabular}

With respect to the algorithm performance, we use the following metrics for the evaluation purpose.

(1) Residual energy: This includes the average residual energy of each node and the energy difference between the node with the most energy and the node with the least energy.

(2) Stability period: The time duration from the network operation until the first node is dead.

(3) The number of data packets received by BS. 


\subsection{Data transmission phase}

Fig. 1 shows the data transmission between all nodes at 1000th and the 1400th round, respectively. The $\mathrm{CMs}$ first send their sensed information to the corresponding $\mathrm{CH}$, and the $\mathrm{CH}$ forwards the information to the BS after aggregating the received data. Blue lines are representing the communication between $\mathrm{CMs}$ and the corresponding $\mathrm{CH}$, while red lines are indicating the transmission of data from $\mathrm{CHs}$ to the BS. As can be seen from the figure, some nodes do not send the sensed information to the corresponding $\mathrm{CH}$; instead they send it directly to the base station. In this way, the transmission distance can be reduced and the CHs' energy consumption can be reduced as well. In Fig. 1(b), some nodes die in the 1400th round, thus, there is no longer any transmission of information between them and other nodes. The transmission between the $\mathrm{CH}$ and the $\mathrm{BS}$ is always single hop communication.

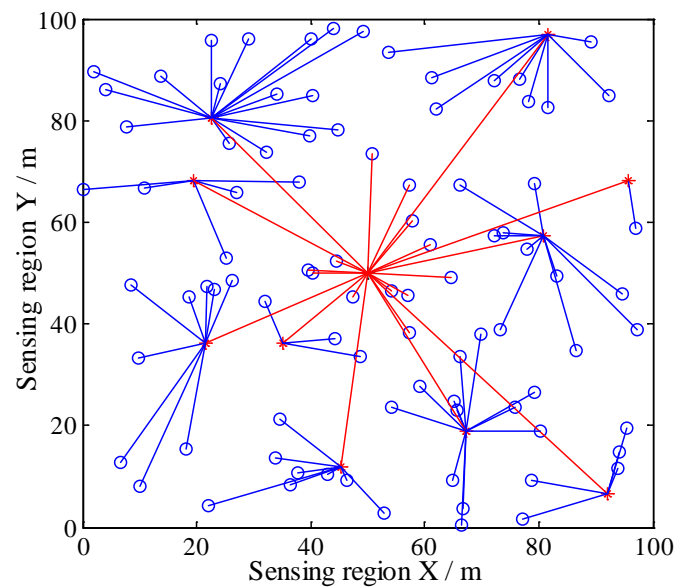

(a) round $=1000$

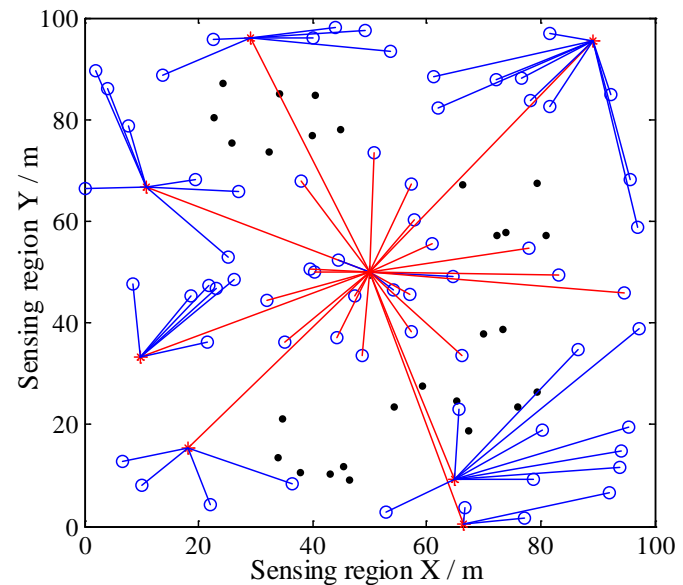

(b) round $=1400$

Fig. 1. Data transmission between all nodes at different rounds

\subsection{Energy distribution}

Fig. 2 and Fig. 3 represent the energy distribution of all nodes at the $1000^{\text {th }}$ and the $1400^{\text {th }}$ round for the three different algorithms. The number near the nodes indicates the residual energy. It can be seen that the nodes' residual energy of the FIGWO algorithm is generally greater than that of the other two algorithms at the same round. In Fig.2, at the $1000^{\text {th }}$ round, the SEP algorithm has 1 dead node, and LEACH has 16 dead nodes, while the remaining energy of all nodes in FIGWO algorithm are all greater than 24\%. In Fig.3, at the $1400^{\text {th }}$ round, the nodes' energy of SEP and LEACH algorithm are almost less than 5\%, however, half of the nodes' energy int the FIGWO algorithm still have more than $10 \%$ of the initial energy. Thus, benefit from the optimal selection of $\mathrm{CH}$ in each round, FIGWO maintains a higher residual energy. 


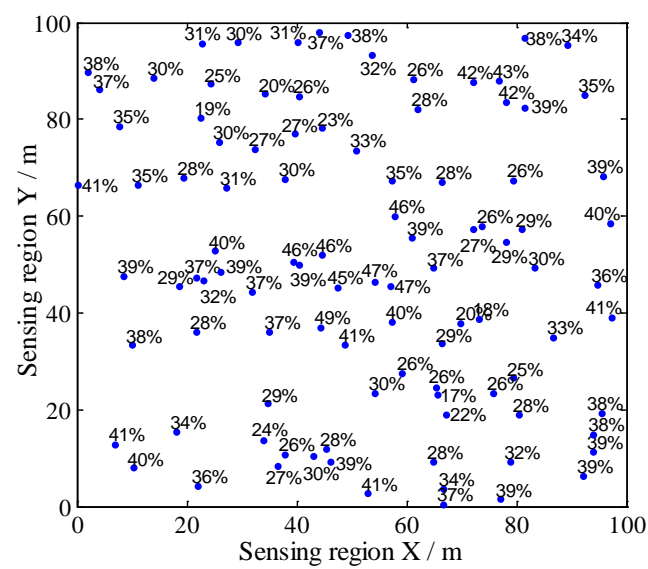

(a) FIGWO, round $=1000$

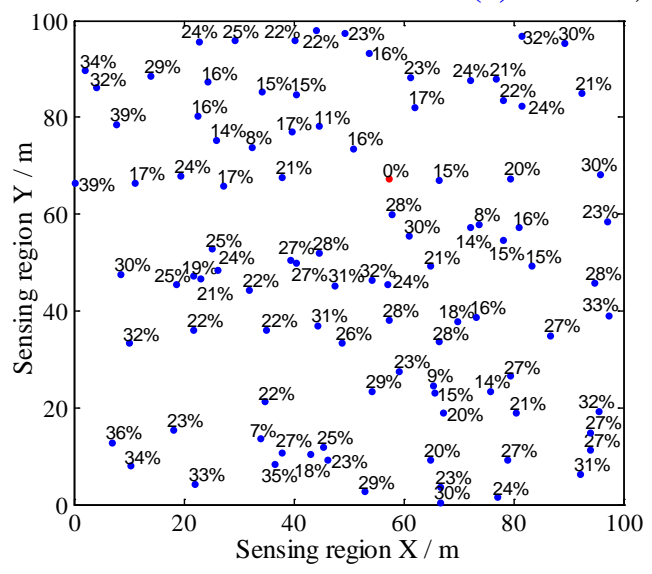

(b) SEP, round $=1000$

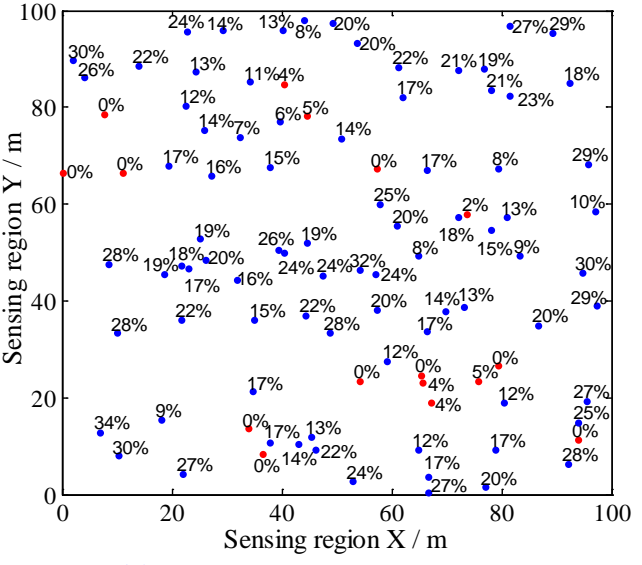

(c) $\mathrm{LEACH}$, round $=1000$

Fig. 2. Energy distribution at 1000 rounds

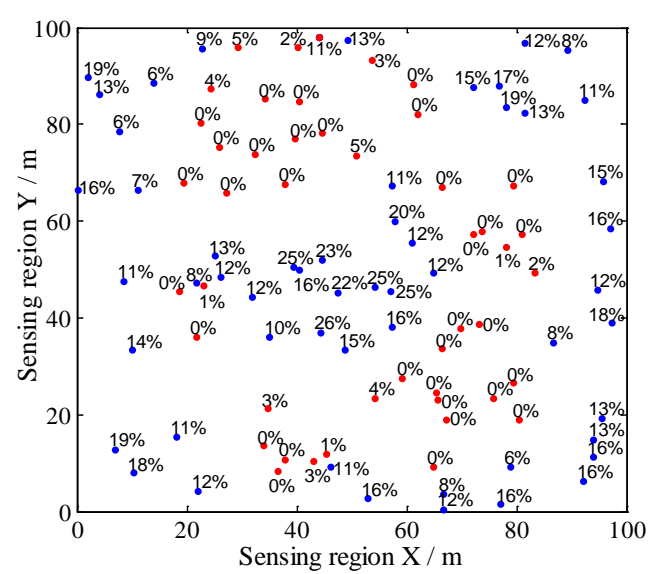

(a) FIGWO, round $=1400$ 


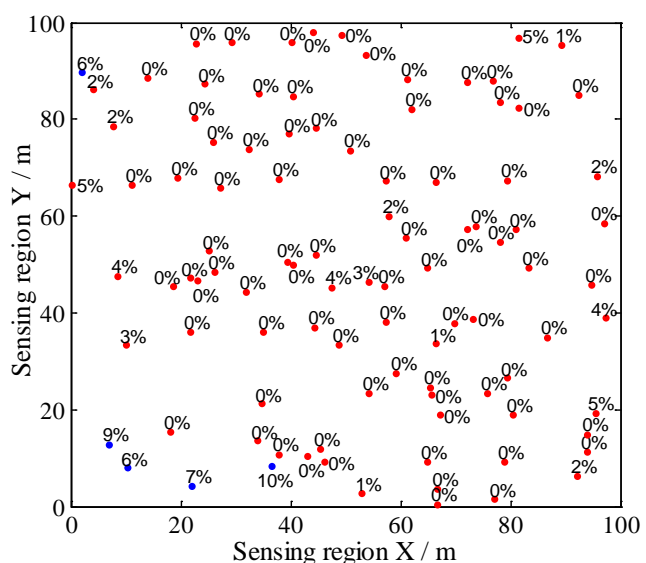

(b) SEP, round $=1400$

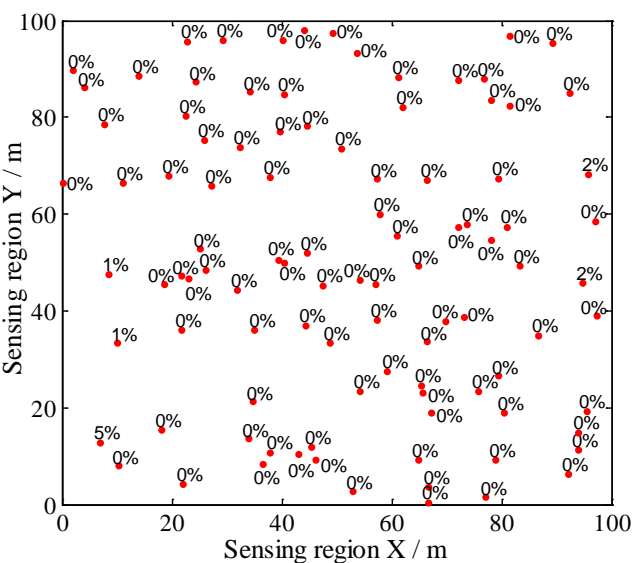

(c) $\mathrm{LEACH}$, round $=1400$

Fig. 3. Energy distribution at 1400 rounds

\subsection{Residual energy}

We also compared the residual energy in more details and for different rounds. This section consists of two parts. Fig. 4(a) and Fig. 4(b) show the average residual energy for each node, while Fig. 4(c) and Fig. 4(d) present the residual energy deviation between the node with the most energy and the node with the least energy. It is obvious that FIGWO achieves a higher average residual energy, which means the network can survive longer. Additionally, the smaller energy deviation illustrates that FIGWO can effectively reduce the energy deviation. There is no such a node that dies prematurely because of excessive energy consumption. Therefore, for the both scenarios, the energy consumption between the nodes using FIGWO is more balanced and due to additional energy savings, the network lifetime is increased in comparison to the other two algorithms.

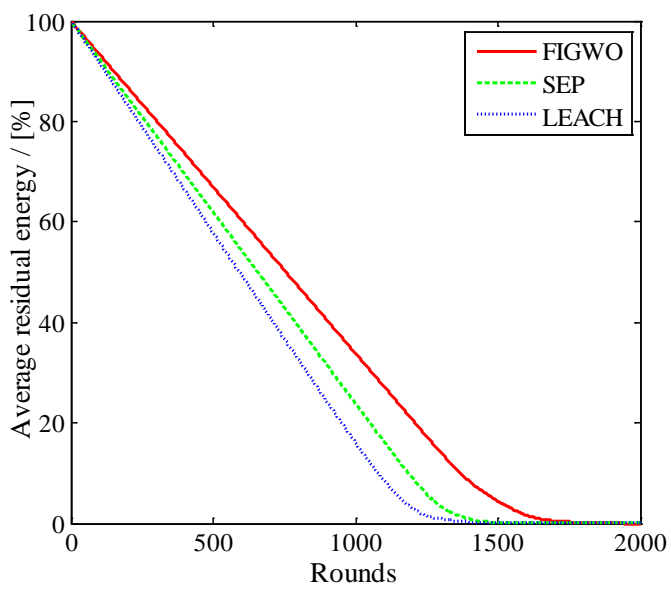

(a) Average residual energy $(\mathrm{N}, \mathrm{M})=\left(100,100^{2}\right)$

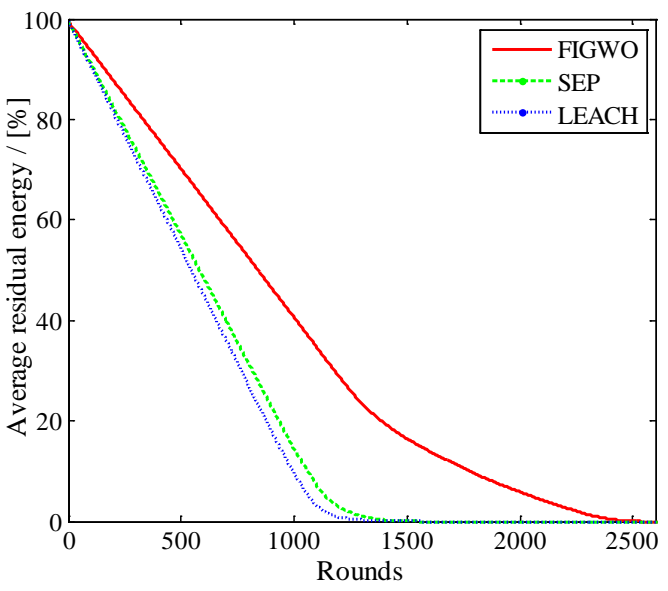

(b) Average residual energy $(\mathrm{N}, \mathrm{M})=\left(200,200^{2}\right)$ 


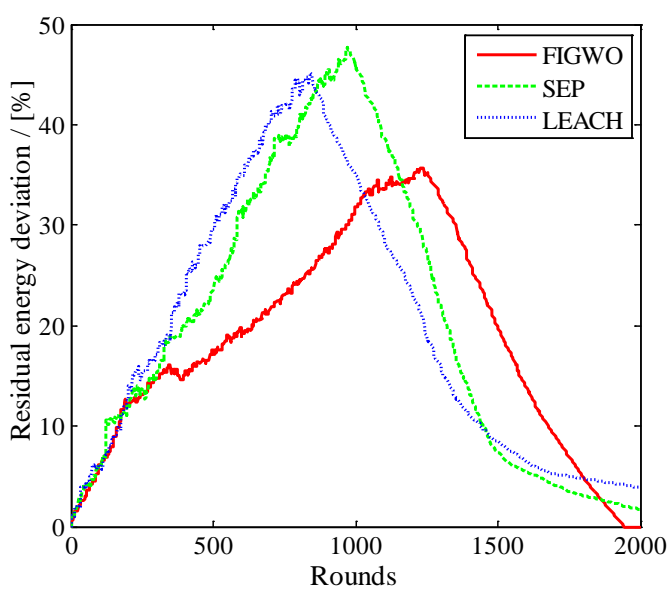

(c) Energy deviation $(\mathrm{N}, \mathrm{M})=\left(100,100^{2}\right)$

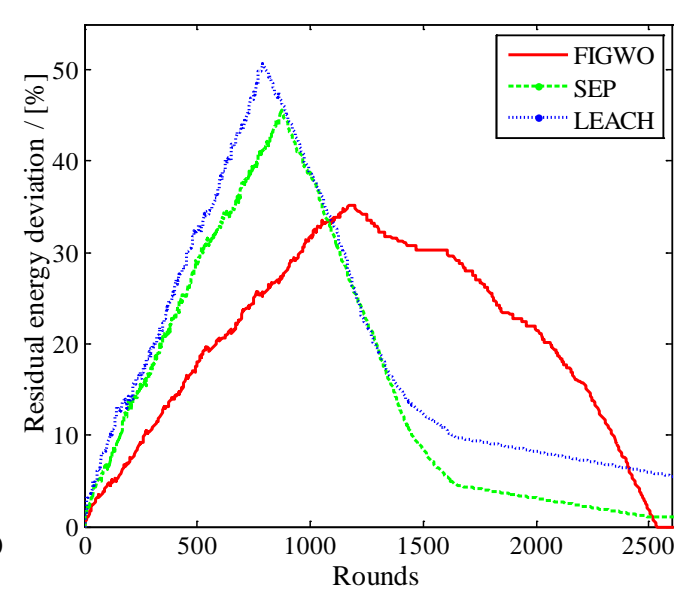

(d) Energy deviation $(\mathrm{N}, \mathrm{M})=\left(200,200^{2}\right)$

Fig. 4. Residual energy with respect to the number of rounds

\subsection{Stability period}

Fig. 5 shows the number of dead nodes for different rounds obtained from the simulation. The network stability period is one of the most important metric in WSNs. It is clearly that FIGWO has more surviving nodes over time than other algorithms, which also indicates that FIGWO has a longer stability period and is able to ensure a stable transmission of sensed data. Because of the more balanced distribution of clusters, FIGWO effectively improves the unbalanced network load and prolongs the stability period.

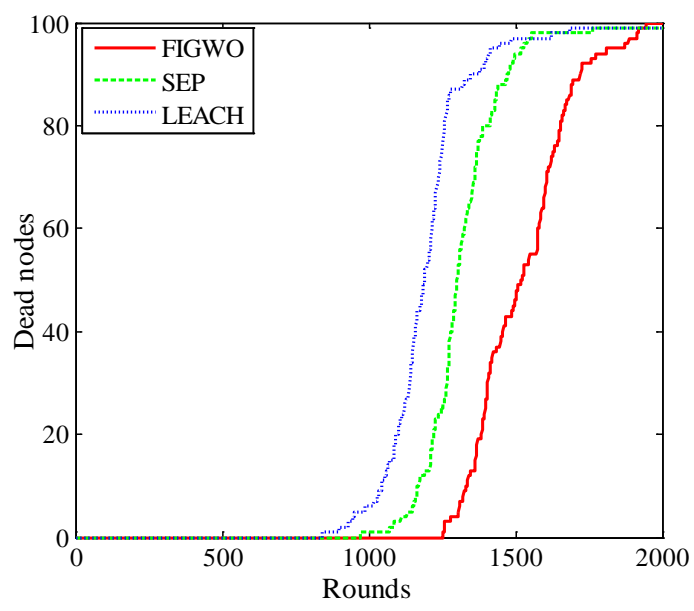

(a) Network stability period $(\mathrm{N}, \mathrm{M})=\left(100,100^{2}\right)$

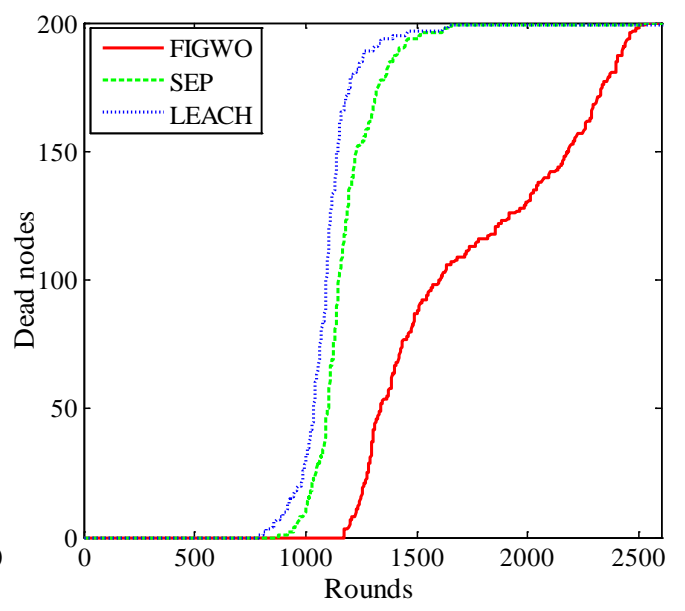

(b) Network stability period $(\mathrm{N}, \mathrm{M})=\left(200,200^{2}\right)$

Fig. 5. Stability period with respect to number of rounds

In Table 5, the concrete values of death rates are shown. From the results, we can conclude that FIGWO has longer stability period for data transmission. It is evident from the table that the stability period is increased by $31.5 \%$ and $57.8 \%$ in scenario 1 and $32.7 \%$ and $48.5 \%$ in scenario 2 in comparison to SEP and LEACH, respectively. 
Table 5. Comparison of network stability period; FND:= Round first nodes dies; HND:=Round half nodes dies; LND:= Round last node dies

\begin{tabular}{|c|c|c|c|c|c|c|}
\hline \multirow{2}{*}{ Algorithm } & \multicolumn{3}{|c|}{$(\mathrm{N}, \mathrm{M})=\left(100,100^{2}\right)$} & \multicolumn{3}{|c|}{$(\mathrm{N}, \mathrm{M})=\left(200,200^{2}\right)$} \\
\hline & FND & HND & LND & FND & HND & LND \\
\hline FIGWO & 1270 & 1510 & 2003 & 1172 & 1612 & 2536 \\
\hline SEP & 966 & 1305 & 1751 & 879 & 1150 & 1857 \\
\hline LEACH & 805 & 1210 & 1982 & 789 & 1093 & 1644 \\
\hline
\end{tabular}

\subsection{The number of data packets received by BS}

For our evaluation, the number of data packets received by BS is another necessary metric. Fig. 6 shows the data delivery ratio to BS. It is clearly that the throughput of the FIGWO algorithm is higher than that of LEACH and SEP. It can also be observed from the figure that the throughput is higher for $144.6 \%$ than in SEP and $177.2 \%$ than in LEACH. This is because some nodes directly send the sensed data to the BS instead of sending it to its $\mathrm{CH}$ first. This reduces the energy consumption while also increasing the total amount of data received by the BS.

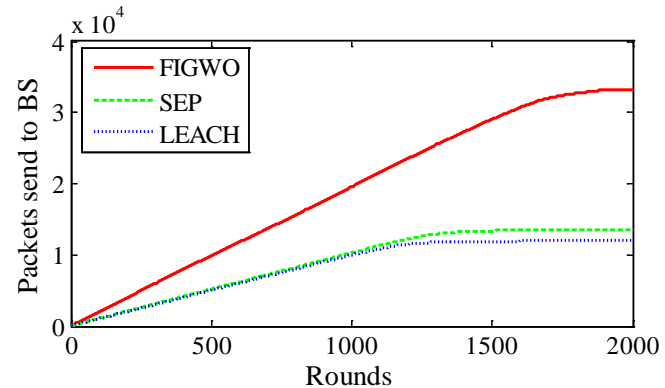

(a) Throughput, $(\mathrm{N}, \mathrm{M})=\left(100,100^{2}\right)$

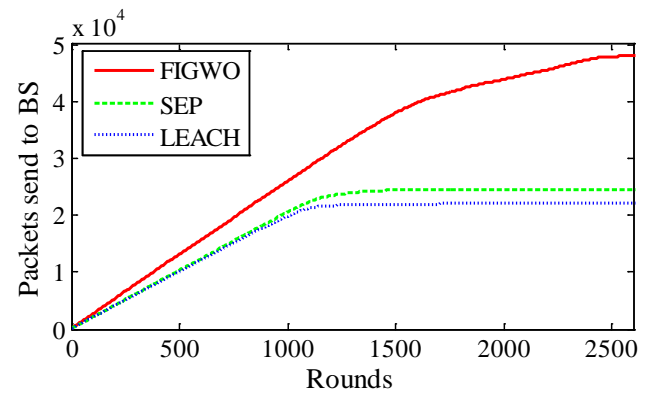

(b) Throughput, $(\mathrm{N}, \mathrm{M})=\left(200,200^{2}\right)$

Fig. 6. The number of data packets received by BS with respect to number of rounds

\section{Conclusion}

In the paper, we proposed a new algorithm for wireless sensor networks (WSNs) with an improved performance in comparison to the standard Grey Wolf Optimization (GWO) algorithm. The algorithm is referred to as Fitness value based Improved Gray Wolf Optimization (FIGWO). It optimizes the method for selection of cluster heads (CHs) by computing a fitness value, thereby ensuring that the node in the cluster located near to the BS and having highest energy is more probably selected as a $\mathrm{CH}$. At the same time, the transmission distance for each node is recalculated any time when new $\mathrm{CH}$ is selected. In this way, both the average transmission distance and the energy consumption are reduced. Consequently, the lifetime of the network can be prolonged.

Our simulation results showed improved performance of the proposed algorithm when compared to the two classical algorithms SEP and LEACH. The performance is improved in terms of the residual energy, stability period, and the amount of data received by BS. The average residual energy of the proposed algorithm is significantly higher and the residual energy deviation is clearly lower than that of the above mentioned algorithms. In addition, FIGWO provides an improved stability period, namely an improvement of $31.5 \%$ with respect to SEP and $57.8 \%$ in comparison to LEACH, which increases the reliability of the data. The throughput of the network is also increased when compared with the two considered 
algorithms.

\section{References}

[1] S. Ehsan and B. Hamdaoui, "A Survey on Energy-Efficient Routing Techniques with QoS Assurances for Wireless Multimedia Sensor Networks," IEEE Communications Surveys \& Tutorials, vol 14, no.2, pp. 265-278, 2012. Article (CrossRef Link)

[2] S. S. Wang and Z. P. Chen, "LCM: A Link-Aware Clustering Mechanism for Energy-Efficient Routing in Wireless Sensor Networks," IEEE Sensors Journal, vol 13, no.2, pp. 728-736, 2013. Article (CrossRef Link)

[3] N. Wang, Y. Zhou, and W. Xiang, "An Energy Efficient Clustering Protocol for Lifetime Maximization in Wireless Sensor Networks," in Proc. of IEEE Conf. on Global Communications(GLOBECO), pp. 1-6, Dec. 4-8, 2016. Article (CrossRef Link)

[4] M. M. Afsar and M. H. Tayarani-N, "Clustering in sensor networks: A literature survey,” Journal of Network \& Computer Applications, vol 46, pp. 198-226, 2014. Article (CrossRef Link)

[5] W. R. Heinzelman, A. Chandrakasan, and H. Balakrishnan, "Energy-Efficient Communication Protocol for Wireless Microsensor Networks," in Proc. of IEEE international Conf. on System Sciences, pp.10, Jan. 7-7, 2000. Article (CrossRef Link)

[6] P. G. Naranjo, M. Shojafar, H. Mostafaei, Z. Pooranian and E. Baccarelli, "P-sep: a prolong stable election routing algorithm for energy-limited heterogeneous fog-supported wireless sensor networks,” Journal of Supercomputing, vol 73 no.2, 733-755, 2017.

Article (CrossRef Link)

[7] S. H. H. Nazhad, M. Shojafar, S. Shamshirband and M. Conti, "An efficient routing protocol for the qos support of large scale MANETs” International Journal of Communication Systems, vol 31, no.2, pp. 1-18, 2017. Article (CrossRef Link)

[8] A. Ray and D. De, "Energy efficient clustering protocol based on K-means (EECPK-means)-midpoint algorithm for enhanced network lifetime in wireless sensor network," IET Wireless Sensor Systems, vol 6, no.6, pp. 181-191, 2016. Article (CrossRef Link)

[9] C. L. Ma, N. Liu and Y. Ruan, "A Dynamic and Energy-Efficient Clustering Algorithm in Large-Scale Mobile Sensor Networks," International Journal of Distributed Sensor Networks, vol 9, no. 11, pp. 1-8, 2013. Article (CrossRef Link)

[10] G. Y. Park, H. Kim, H. W. Jeong and H. Y. Youn, "A Novel Cluster Head Selection Method based on K-Means Algorithm for Energy Efficient Wireless Sensor Network," in Proc. of IEEE International Conf on Advanced Information networking and Applications Workshops(WAINA), pp.910-915, March. 25-28, 2013. Article (CrossRef Link)

[11] A. Ahmadi, M. Shojafar, S. F. Hajeforosh, M. Dehghan and M. Singhal, "An efficient routing algorithm to preserve k-coverage in wireless sensor networks," Journal of Supercomputing, vol. 68, no. 2, pp. 599-623, 2014. Article (CrossRef Link)

[12] N. A. Al-Aboody and H. S. Al-Raweshidy, "Grey wolf optimization-based energy-efficient routing protocol for heterogeneous wireless sensor networks," in Proc. of IEEE International Symposium on Computational and Business Intelligence(ISCBI), pp. 101-107, Sept. 5-7, 2016. Article (CrossRef Link)

[13] H. Mostafaei and M. Shojafar, "A New Meta-heuristic Algorithm for Maximizing Lifetime of Wireless Sensor Networks," Kluwer Academic Publishers, vol 82, no. 2, pp. 723-742, 2015. Article (CrossRef Link)

[14] S. Mirjalili, S. M. Mirjalili, and A. Lewis, "Grey Wolf Optimizer," Advances in Engineering Software, vol 69, no.3, pp. 46-61, 2014. Article (CrossRef Link) 


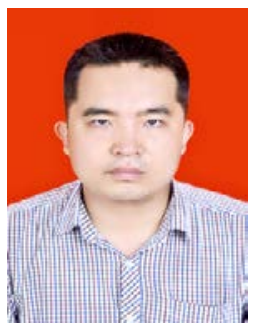

Xiaoqiang Zhao is now a professor in Xi'an University of Posts and Telecommunications, Xi'an, China. He received the Ph.D. degree from Xi'an University of Technology, Xi'an, China, in 2015. His current research interests include Agricultural Internet of Things Technology and Environmental Engineering.

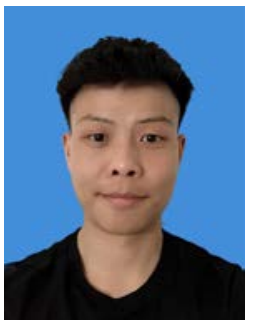

Hui Zhu is currently pursuing the master degree in Xi'an University of Posts and Telecommunications, Xi'an, China. His research interests include wireless sensor network protocol, digital signal processing.

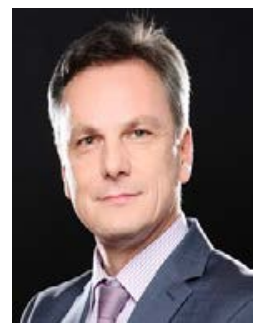

Slavisa Aleksic is a full professor at the Leipzig University of Telecommunications, Germany. He received both the M.Sc. and Ph.D. degrees from Vienna University of Technology in 1999 and 2004, respectively. His research interests include telecommunication systems, network technologies, as well as network design and evaluation.

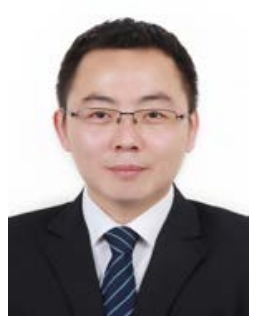

Qiang Gao is currently pursuing the master degree in Xi'an University of Posts and Telecommunications, Xi'an, China. His research interests include digital signal processing, automatic control system. 